\title{
Trypanosomosis in Goats
}

\section{Current Status}

\section{CARLOS GUTIERREZ, ${ }^{a}$ JUAN A. CORBERA,${ }^{a}$ MANUEL MORALES,${ }^{a}$ AND PHILIPPE BÜSCHER ${ }^{b}$}

${ }^{a}$ Veterinary Faculty, University of Las Palmas, Las Palmas, 35416 Canary Islands, Spain

${ }^{b}$ Institute of Tropical Medicine, Nationalstraat 130, Antwerp, Belgium

\begin{abstract}
Trypanosomosis is a major constraint on ruminant livestock production in Africa, Asia, and South America. The principal host species affected varies geographically, but buffalo, cattle, camels, and horses are particularly sensitive. Natural infections with Trypanosoma congolense, $T$. vivax, T. brucei, and $T$. evansi have been described in goats. Trypanosomosis in goats produces acute, subacute, chronic, or subclinical forms, being $T$. vivax, $T$. congolense, and $T$. evansi, the most invasive trypanosomes for goats. However, the role of goats in the epidemiology of trypanosomosis is largely discussed and not well understood. Thus, it has commonly been assumed that trypanosomosis presents a subclinical course and that goats do not play an important role in the epidemiology of the disease. This can partially be due to parasitemia caused by trypanosomes which has been considered low in goats. However, this assumption is currently undergoing a critical reappraisal because of goats may also serve as a reservoir of trypanosome infection for other species, including the human beings in the case of $T$. brucei rhodesiense. The present article describes the current status of trypanosomosis in goats in Africa, Asia, and South America. Pathogenesis, clinical features, diagnosis, and treatment of the different trypanosomes are also described. The possible role in the epidemiology of the disease in the different areas is also discussed.
\end{abstract}

KeYWORDs: Trypanosoma spp.; trypanosomosis; goats; current status

\section{INTRODUCTION}

Trypanosomosis is a major constraint on ruminant livestock production in many areas of Africa, Asia, and South America. Many animal species can be

Address for correspondence: Carlos Gutierrez, Veterinary Faculty, University of Las Palmas, Arucas, Las Palmas, 35416 Canary Islands, Spain. Voice: 34-928451115; fax: 34-928451142.

e-mail: cgutierrez@dpat.ulpgc.es

Ann. N.Y. Acad. Sci. 1081: 300-310 (2006). (C) 2006 New York Academy of Sciences. doi: 10.1196/annals.1373.040 
affected by the different trypanosomes, thus severely impairing the economic efficiency in endemic areas.

Trypanosoma are classically divided into Stercoraria and Salivaria sections, according to their life cycle in the insect vector. Within the Salivaria section, some species (e.g., T. congolense and T. brucei) are transmitted only by tsetse flies (Glossina spp.) and are called the tsetse-transmitted trypanosomes. Others may be either cyclically transmitted by tsetse flies, or mechanically transmitted by other hematophagous insects (e.g., T. vivax). Still others are only mechanically (T. evansi) or sexually (T. equiperdum) transmitted (TABLE 1).

Tsetse-transmitted trypanosomosis is a disease complex caused by several species of Trypanosoma. The many species of tsetse infest 10 million square kilometers and affect 37 countries in Africa. The disease affects both humans and livestock. It is currently estimated that about 50 million people ${ }^{1}$ and 48 million cattle ${ }^{2}$ are at risk of contracting trypanosomosis. From an economic point of view, the disease is particularly important in cattle, although other mammals can also be affected. The livestock-carrying capacity of such areas in West and Central Africa could be increased five- to sevenfold by eliminating or controlling animal trypanosomosis. ${ }^{3}$

Trypanosomosis caused by T. evansi (surra) is the most widely distributed of the pathogenic animal trypanosomes affecting livestock in South America, Africa, and Asia. The most affected animal species are buffalo, horses, camels, and cattle, although geographical differences are observed. Other hosts, including small ruminants, can be affected.

There are approximately 228 million goats in $\mathrm{Africa}^{4}$ (data on Somalia are not available) with as many as 173 million in the tsetse-infested regions of the continent.

It is commonly believed that goats are highly resistant to infection, that caprine trypanosomosis is only sporadic, and that the disease in goats is of little economic consequence. ${ }^{3}$ However, current epidemiological information indicates that goats can play an important role in the dissemination of the disease. Thus, goats naturally infected with $T$. congolense, $T$. vivax, or T. brucei and presenting clinical disease are regularly observed in Africa. Regional differences in the prevalence of caprine trypanosomosis exist, but can be high in some areas. ${ }^{5,6}$ In general, caprine trypanosomosis is more common in East than in West Africa. This has been attributed to differences in feeding preferences between riverine and savannah species of Glossina; the latter being more inclined to feed on goats. ${ }^{7}$

Trypanotolerance has clearly been established in cattle, particularly in the breeds N'dama of West Africa and West African Shorthorn. The existence of trypanotolerance in certain goat and sheep breeds is under study. Dwarf goats are known to be more resistant to trypanosomosis than exotic breeds or breeds living in tsetse-free areas. ${ }^{5,8}$ Studies carried out by Goossens et al. ${ }^{9}$ seem to demonstrate that African Dwarf goats are less trypanotolerant than Djallonke 


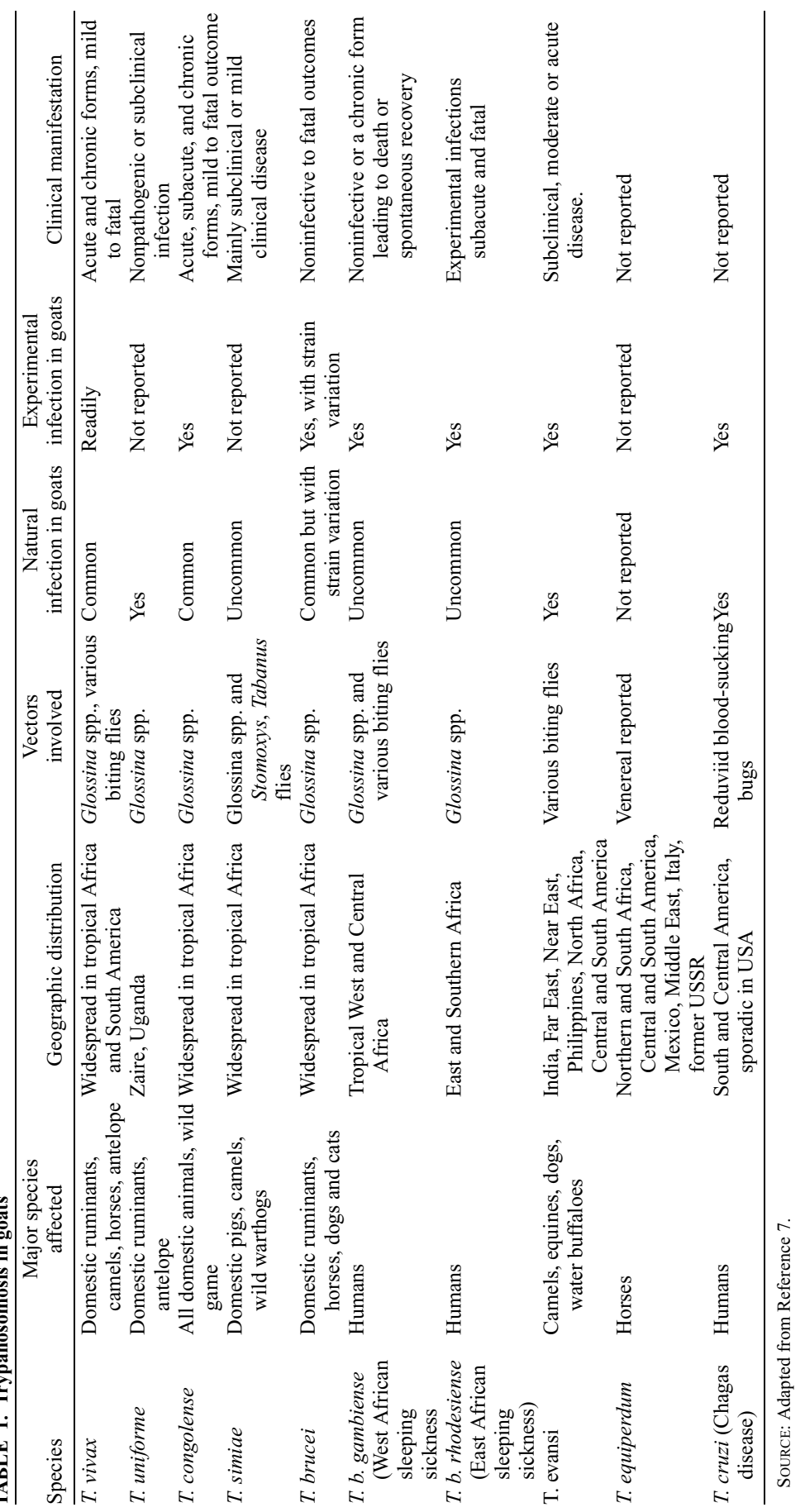


sheep. The trypanotolerance in cattle has been defined as their ability to control parasitemia and anemia. ${ }^{10}$ However, small ruminant are less able to control parasitemia and drop in packed cell volume (PCV) following trypanosome infection. ${ }^{11}$ On the other hand, Murray et al. ${ }^{12}$ described that Glossina flies may select other livestock over goats when mixed animal populations are present, which can contribute to survival in tsetse areas. The studies carried out in goats seem to be insufficient to understand the true mechanism of trypanotolerance in this species.

Regarding treatment, drug resistance has become a significant problem because no new drugs have been marketed for quite some time. In general, curative doses used in cattle are also appropriate for goats and sheep..$^{13}$ Diminazene aceturate is given intramuscularly as a $7 \%$ water solution at a dose of 3.5 $\mathrm{mg} / \mathrm{kg}$; quinapyramine dimethyl sulfate given intramuscularly as a $10 \%$ water solution at a dose of $10.0 \mathrm{mg} / \mathrm{kg}$. Both are considered as very effective. Relapse of infection has been reported in goats treated with diminazene aceturate, presumably because of re-emergence of trypanosomes from the central nervous system where they were inaccessible to the drug during earlier treatment. ${ }^{8}$

Homidium chloride or homidium bromide are given in a $2 \%$ water solution at a dose of $1.0 \mathrm{mg} / \mathrm{kg}$ intramuscularly, both are effective against $T$. vivax and $T$. congolense. Isometamidium chloride is also effective against the trypanosomes in the blood when given at a dose of $0.25-0.75 \mathrm{mg} / \mathrm{kg}$ intramuscularly as a $1 \%$ or $2 \%$ water solution. This drug was shown to produce signs of shock or death in goats if given intravenously at doses greater than or equal to $0.5 \mathrm{mg} / \mathrm{kg} .{ }^{14}$ Goats experimentally infected with $T$. evansi were cured after a single inoculation of $0.3 \mathrm{mg} / \mathrm{kg}$ of an arsenal compound (Cymelarsan ${ }^{\circledR}$; Merial, Lyon, France)..$^{15}$

In many areas in the world, goats are reared near or in contact to highly susceptible species (particularly cattle, dromedaries, and horses). However, their role as possible reservoirs and carriers of the trypanosomes is largely discussed. In this article, we expose the current knowledge of trypanosomosis in goats.

\section{T. EVANSI}

T. evansi is the causative agent of surra, a disease of camel and horses that produces important economic losses in endemic areas (FIGS. 1-3). The disease has been described in small ruminants causing subclinical infection, ${ }^{16-18}$ moderate infection, ${ }^{19}$ and severe infection. ${ }^{19,20}$ Ngeranwa et al. ${ }^{19}$ described erratic parasitemia, weight loss, and significant drop in PCV in an experimental inoculation using a T. evansi strain isolated in Kenya and inoculating small East African bucks. Natural infection has been described in goats by serology ${ }^{21,22}$ in a survey carried out in Sudan. However, goats kept in contact with parasitemic camels and horses were found aparasitemic in Jordan. ${ }^{23}$ To 


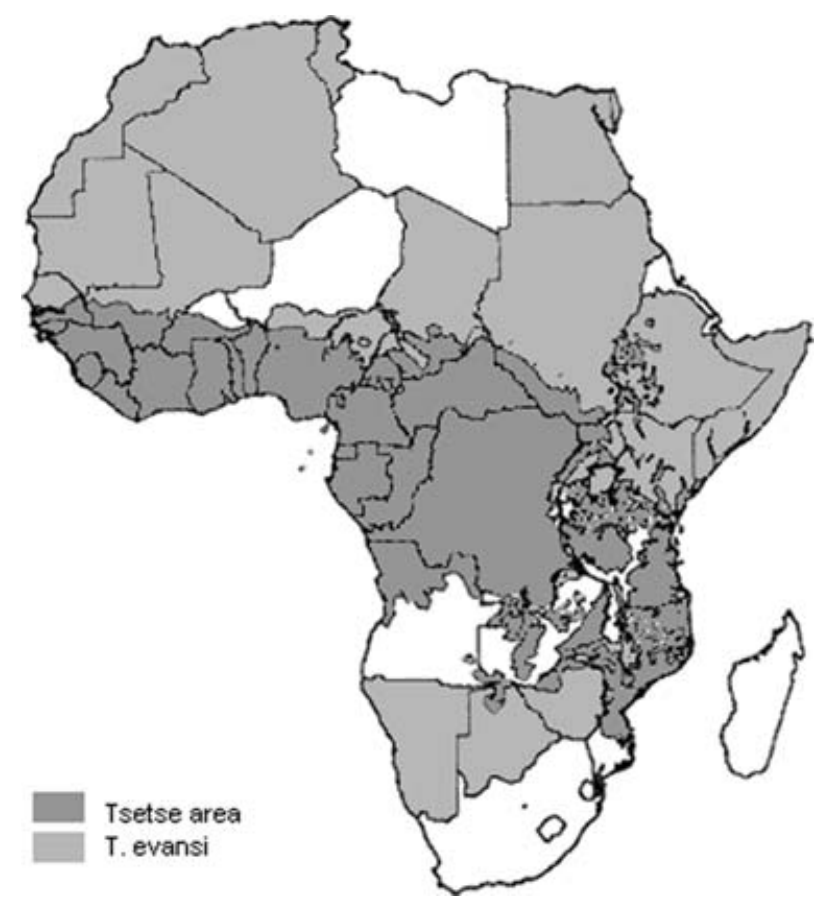

FIGURE 1. Tropical area in Africa (tsetse area) as well as countries where T. evansi has been reported.

isolate T. evansi from affected goats is very difficult due to low parasitemia. Ngeranwa et al. ${ }^{19}$ observed a predominant presence of $T$. evansi in extravascular locations (synovial, peritoneal and cerebrospinal fluids, and lymph nodes). T. evansi can pass to blood from these extravascular locations in febrile or advances stages in goats. Common parasitological detection tests for Trypanosoma spp. employed in other animal species are also valid for goats, including the mini anion exchange centrifugation technique. ${ }^{24}$

The small size of the caprine erythrocytes $(3.2 \mu \mathrm{m}$; for comparison: 6.5 $\mu \mathrm{m}$ for camels, $5.5 \mu \mathrm{m}$ for horses, and $5.8 \mu \mathrm{m}$ for cattle) must be taken into consideration for obtain a valid PCV (Woo technique ${ }^{25}$ ). A mean cell hemoglobin concentration (MCHC) around $30-36 \%$ is considered a proper centrifugation for normal goat blood. ${ }^{26}$

Indirect methods seem to be more appropriate to detect T. evansi infections in goats. An enzyme-linked immunosorbent assay (ELISA) for the detection of $T$. evansi in goat sera using a monoclonal antibody has been reported. ${ }^{27}$ Indirect immunofluorescence (IFI) has also been used to detect $T$. evansi antibodies in goats. ${ }^{28} \mathrm{~A}$ direct card agglutination test (CATT/T. evansi) and an indirect latex agglutination test (LATEX/T. evansi) have been shown adequate to detect T. evansi antibodies in goat sera. ${ }^{29}$ 


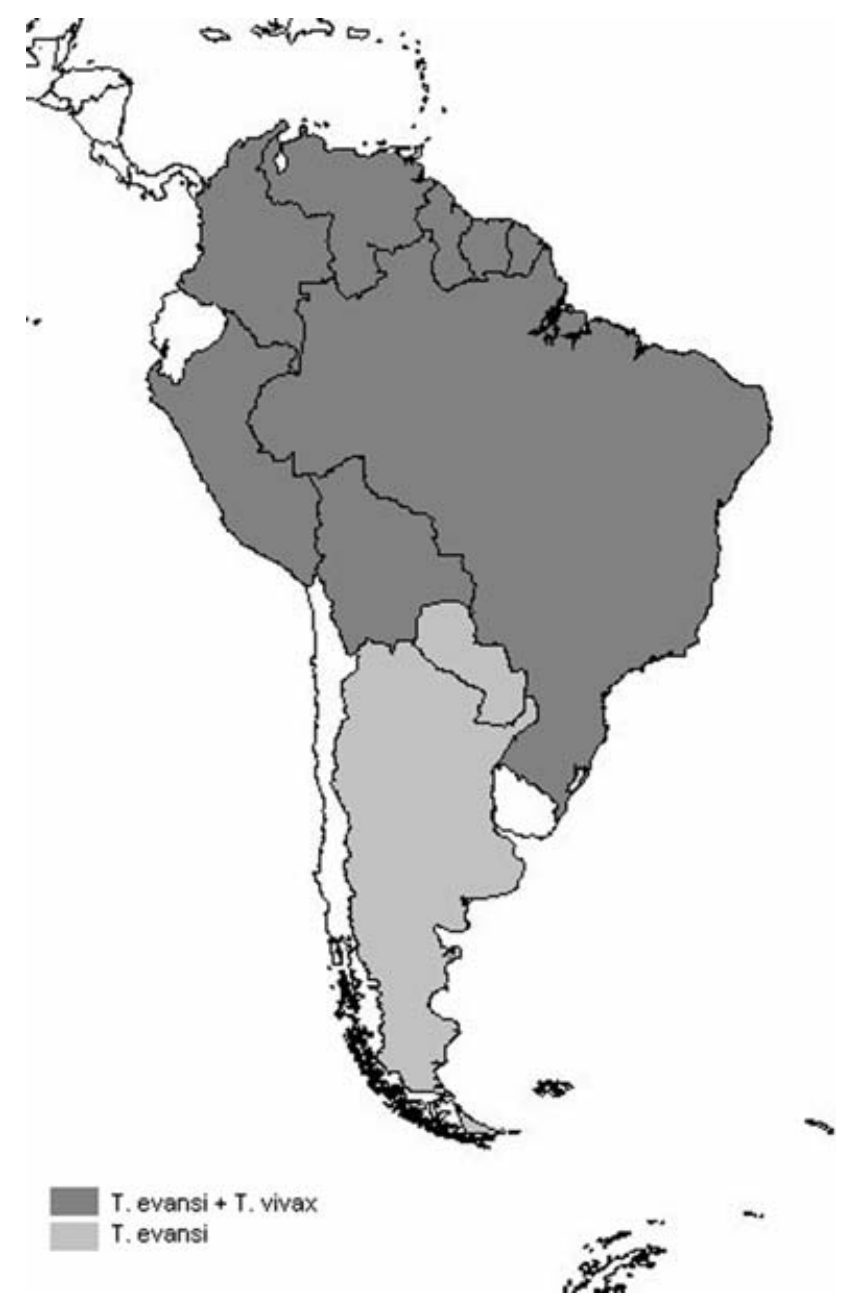

FIGURE 2. Presence of T. evansi and T. vivax in South America based on Dávila and Silva. $^{41}$

\section{T. CONGOLENSE}

T. congolense is the most common trypanosome of goats in Africa. Goats can also act as a reservoir of $T$. congolense for other species. In the Sudan, goats infected with $T$. congolense developed a chronic form of disease from which many spontaneously recovered. When the organism was passaged from goats into calves, however, acute fatal bovine trypanosomosis occurred. ${ }^{18}$

The trypanotolerance to T. congolense of West African Dwarf goats is similar to its F1 crosses with the Sahelian breed (trypanosusceptible). ${ }^{30}$ Similar results have also been reported by Dhollander et al. ${ }^{31}$ studying West African Dwarf goats and its F1 crosses with Saanen goats (trypanosusceptible). The African 


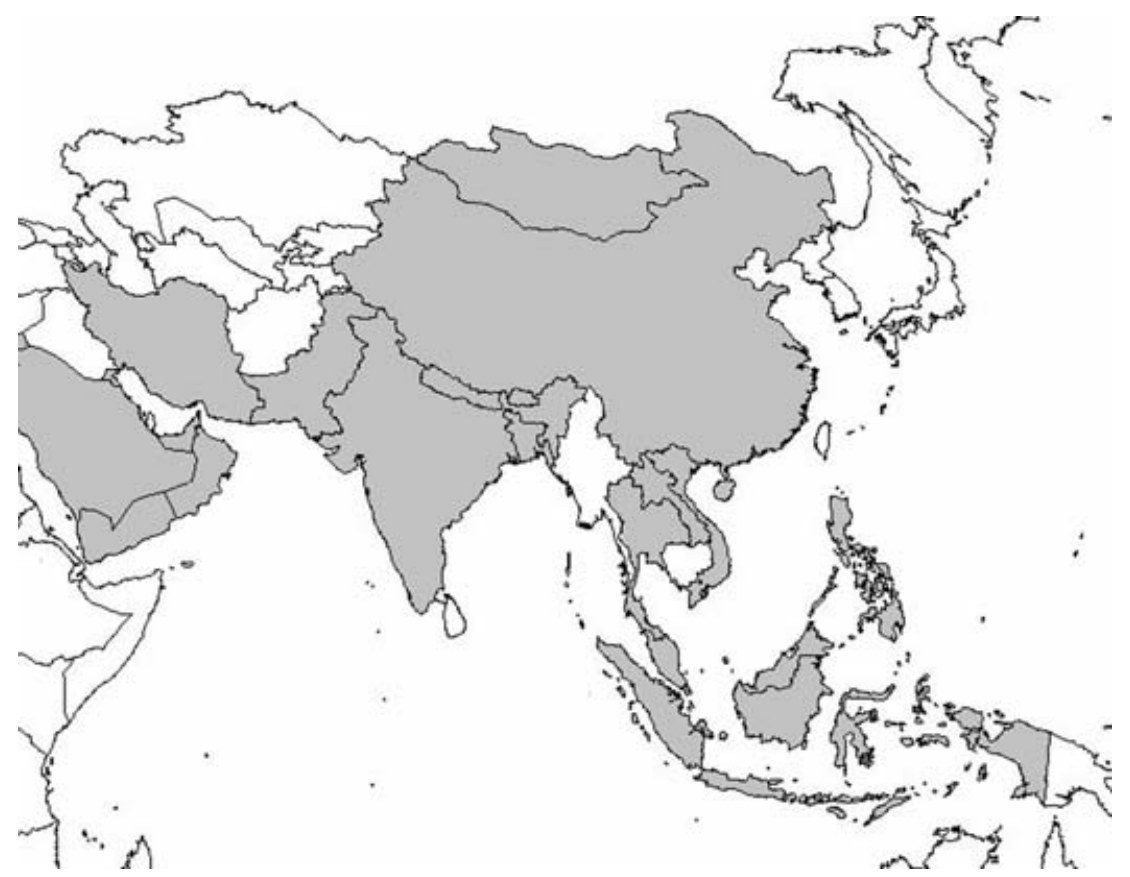

FIGURE 3. Asia. Countries in which T. evansi has been described.

gray duiker (Sylvicapra grimmia) is also considered as trypanotolerant, even much more than sheep and goats. ${ }^{32}$

T. congolense produces immunosuppressive effects in West African Dwarf goats infected simultaneously with Haemonchus contortus. ${ }^{30}$ Experimental T. congolense infection in the same breed affected reproductive performance with abortions, premature births, and perinatal losses. Transplacental transmission of the parasite or lesions in placenta could not be demonstrated. ${ }^{33}$ In another experimental inoculation of $T$. congolense in goats, Witola and Lovelace $^{34}$ indicated that erythrophagocytosis by mononuclear cells is a mechanism of anemia pathogenesis.

From the diagnosis point of view, detection of $T$. congolense DNA by polymerase chain reaction (PCR) using GOL as primer set was $100 \%$ concordant with buffy coat examination. ${ }^{22}$ Also, an indirect ELISA to detect $T$. congolense antibodies in goats has been described by Lejon et al. ${ }^{35}$

\section{T. BRUCEI}

Natural infection of goats with $T$. brucei is sporadically reported. A survey carried out in Gambia showed that $T$. brucei was present in West African Dwarf goats but only in a few cases. ${ }^{36}$ From the experimental viewpoint, 
T. brucei infection has been reproduced in Nigerian West African Dwarf goats using a local virulent strain. ${ }^{37}$ In an immunity study, infection of goats with T. brucei resulted in depressed responses to mitogen stimulation of lymphocyte transformation. ${ }^{38}$

Goats and other domestic animals are relatively resistant to T. brucei gambiense, which is the cause of West African human sleeping sickness; however, goats have been suggested as reservoir host of the parasite. ${ }^{39}$ When infection does occur, the clinical course is chronic. T. brucei rhodesiense is an uncommon cause of caprine disease. ${ }^{7}$ Goats also have been implicated as a reservoir of $T$. brucei rhodesiense, transmissible to man. ${ }^{40}$

\section{T. VIVAX}

T. vivax is the second most common trypanosome of goats in Africa. T. vivax can be transmitted to domestic livestock by tsetse flies (Glossina spp., cyclical transmission) as well as directly (mechanical transmission) by other bloodsucking insects thus allowing T. vivax to extend its distribution beyond tropical Africa. T. vivax was introduced in South America in 1830 by a shipment of zebu cattle from Senegal. ${ }^{41}$ Today, goats are recognized as reservoir host for T. vivax in many countries in South America. ${ }^{42}$

T. vivax produces immunosuppression, which has also been demonstrated in goats. ${ }^{43}$ Thrombocytopenia, microthrombus formation and hemorrhage suggestive of disseminated intravascular coagulation (DIC) have been observed in goats infected with T. vivax. ${ }^{44,45}$

\section{T. CRUZI}

Natural infection of T. cruzi in goats has been detected by Herrera et al ${ }^{46}$ using indirect fluorescent antibody test. Clinical manifestation of the disease has not been reported. Kids experimentally infected with $T$. cruzi showed no clinical signs of disease and carried the infection for 38 days. ${ }^{47}$ Regarding the epidemiology of the disease in a Brazilian endemic area of Chagas' disease, Herrera et al. ${ }^{46}$ described that goats did not play an apparent role.

\section{OTHER TRYPANOSOMES}

Goats are susceptible to T. uniforme, in Uganda and Zaire, but only mild infections occur. T. simiae, a trypanosome of swine and camels is transmissible to goats by either Glossina spp. or by biting flies but causes mostly mild or subclinical disease. ${ }^{7}$

A nonpathogenic trypanosome, T. theodori, was found incidentally in goats in Israel. ${ }^{7}$ It is transmitted by a hippoboscid fly, Lipoptena caprina. This 
organism is morphologically similar to the common, nonpathogenic sheep trypanosome, T. melophagium.

In conclusion, goats are highly susceptible to pathogenic trypanosomes, although the disease follows commonly a subclinical course. Parasitemia is usually low but persistent, and therefore, goats can be considered as an important reservoir for the majority of trypanosomes of other animals and humans. In those areas of the world where the presence of goats is important (in desert or semi-desert zones), caprine trypanosomosis should be taken into consideration in all programs to control the disease.

\section{REFERENCES}

1. Kuzoe, F.A.S. 1991. Perspectives in research on control of African trypanosomiasis. Ann. Trop. Med. Parasitol. 85: 33-41.

2. Kristuanson, P.M., B.M. Swallow, G.J. Rowlands, et al. 1999. Measuring the costs of African animal trypanosomosis, the potential benefits to control and returns to research. Agr. System 59: 79-98.

3. GRIFFIN, L. 1978. African trypanosomosis in sheep and goats: a review. Vet. Bull. 48: 819-825.

4. FAO (Food ANd Agriculture Organization). 2004. FAOSTAT. www.fao.org.

5. Grifrin, L. \& E.W. Allonby. 1979. Studies on the epidemiology of trypanosomiasis in sheep and goats in Kenya. Trop. Anim. Health Prod. 11: 133-142.

6. Kramer, J.W. 1966. Incidence of trypanosomes in the West African Dwarf sheep and goat in Nsukka, eastern Nigeria. Bull. Epizoot. Dis. Afr. 14: 423-428.

7. Smith, M.C. \& D.M. Sherman. 1994. Blood, lymph, and immune system. In Goat Medicine. M.C. Smith \& D.M. Sherman, Eds.:193-230. Lea \& Febiger. Philadelphia.

8. Whitelaw, D.D., J.E. Moulton, W.I. Morrison, et al. 1985. Central nervous system involvement in goats undergoing primary infections with Trypanosoma brucei and relapse infections after chemotherapy. Parasitology 90: 255-268.

9. Goossens, B., S. OSAer \& S. Kora. 1997. Long-term effects of an experimental infection with Trypanosoma congolense on reproductive performance of trypanotolerant Djallonké ewes and West African Dwarf does. Res. Vet. Sci. 63: 169-173.

10. Murray, M., W.I. Morrison \& D.D. Whitelaw. 1982. Host susceptibility to African trypanosomiasis: trypanotolerance. Adv. Parasitol. 21: 1-68.

11. Osaer, S., B. Goossens, D.J. Clifford, et al. 1994. A comparison of the susceptibility of Djallonke sheep and West African Dwarf goats to experimental infection with two different strains of Trypanosoma congolense. Vet. Parasitol. 51: 191-204.

12. Murray, M., J.C.M. Trail, C.E. Davis, et al. 1984. Genetic resistance to African trypanosomosis. J. Infect. Dis. 149: 311-319.

13. Ilemobade, A.A. 1986. Tripanosomiasis (nagana, samore, tsetse fly disease). In Current Veterinary Therapy, Food Animal Practice, 2nd ed. J. Howard, Ed.: 642645. WB Saunders. Philadelphia.

14. Schillinger, D., S.H. Maloo \& D. RotTcher. 1985. The toxic effect of intravenous application of the trypanocide isometamidium (samorin). Zbl. Vet. Med. A 32: 234-239. 
15. Zweygarth, E., J. Ngeranwa \& R. Kaminsky. 1992. Preliminary observations on the efficacy of mel Cy (Cymelarsan) in domestic animals infected with stocks of Trypanosoma brucei brucei and T. b. evansi. Trop. Med. Parasitol. 43: 226-228.

16. KhasAnov, L. \& E.D. IvanitskayA. 1974. Experimental trypanosomiasis (T. ninaekolhjakimovi) infection in sheep and goats. Trudy Uzbekskogo Nauchoissledovatelskogo Veterinarnogo Instituta 22: 98-100.

17. Otieno, P.S. \& J.W. Gachanja. 1976. Studies of experimental Trypanosoma evansi infection in goats. Bull. Anim. Health Prod. Afr. 24: 295-297.

18. Mahmoud, M.M. \& K.H. ElmaliK. 1977. Trypanosomiasis: goats as a possible reservoir of Trypanosoma congolense in the Republic of the Sudan. Trop. Anim. Health Prod. 9: 167-170.

19. Ngeranwa, J.J., P.K. Gathumbi, E.R. Mutiga, et al. 1993. Pathogenesis of Trypanosoma (brucei) evansi in small east African goats. Res. Vet. Sci. 54: 283289.

20. Antao, S., D. Steel \& G.M. Warui. 1968. The infectivity of Trypanosoma evansi to some East African mammals. 12th Meeting of the International Scientific Council for Trypanosomiasis. Banjul, The Gambia. Publication number 102, 123-124.

21. Boid, R., E.A. El-Amin, M.M. Mahmoud, et al. 1981. Trypanosoma evansi infections and antibodies in goats, sheep and camels in the Sudan. Trop. Anim. Health Prod. 13: 141-146.

22. Pereira De Almeida, P.J., M. Ndao, B. Goossens, et al. 1998. PCR primer evaluation for the detection of trypanosome DNA in naturally infected goats. Vet. Parasitol. 80: 111-116.

23. Aвo-Shehada, M.N., H. Anshassi, G. Mustafa, et al. 1999. Prevalence of surra among camels and horses in Jordan. Prev. Vet. Med. 38: 289-293.

24. Gutierrez, C., J.A. Corbera, F. Doreste, et al. 2004. Use of miniature anion exchange centrifugation technique to isolate Trypanosoma evansi from goats. Ann. N. Y. Acad. Sci. 1026: 149-151.

25. WoO, P.T.K. 1969. The haematocrit centrifuge for the detection of trypanosomes in blood. Can. J. Zool. 47: 921-923.

26. JAIN, N.C. 1986. Schalm's Veterinary Hematology, 4th Ed. Lea \& Febiger. Philadelphia.

27. Olaho-Mukani, W., W.K. Munyua \& A.R. Nuogu. 1992. An enzyme-linked immunosorbent assay (ELISA) for the detection of trypanosomal antigens in oat serum using a monoclonal antibody. J. Immunoassay 13: 217-229.

28. JACQUiet, P., D. CheIKH, A. ThIAM, et al. 1993. La trypanosomose à Trypanosoma evansi (Steel 1885), Balbiani 1888 chez les petits ruminants de Mauritanie: résultats d'inoculation expérimentale et d'enquetes sur le terrain. Rev. Elev. Méd. Vét. Pays Trop. 46: 574-578.

29. Gutierrez, C., J.A. Corbera, M. Morales, et al. 2004. Performance of serological tests for Trypanosoma evansi in experimentally inoculated goats. Ann N. Y. Acad. Sci. 1026: 152-153.

30. Faye, D., S. Osaer, B. Goossens, et al. 2002. Susceptibility of trypanotolerant West African Dwarf goats and F1 crosses with the susceptible Sahelian breed to experimental Trypanosoma congolense infection and interactions with helminth infections and different levels of diet. Vet. Parasitol. 108: 117-136.

31. Dhollander, S., J. Bos, S. Kora, et al. 2005. Susceptibility of West African Dwarf goats and WAD x Saanen crosses to experimental infection with Trypanosoma congolense. Vet. Parasitol. 130: 1-8. 
32. Ogunsanmi, A.O. \& V.O. Taiwo. 2001. Pathobiochemical mechanisms involved in the control of the disease caused by Trypanosoma congolense in African grey duiker (Sylvicapra grimmia). Vet. Parasitol. 96: 51-63.

33. FAYe, D., J. Sulon, Y. KAne, et al. 2004. Effects of an experimental Trypanosoma congolense infection on the reproductive performance of West African Dwarf goats. Theriogenology 62: 1438-1451.

34. Witola, W.H. \& C.E.A. Lovelace. 2001. Demonstration of erythrophagocytosis in Trypanosoma congolense-infected goats. Vet. Parasitol. 96: 115-126.

35. Lejon, V., D.E. Rebeski, M. NdaO, et al. 2003. Performance of enzyme-linked immunosorbent assays for detection of antibodies against $T$. congolense and T. vivax in goats. Vet. Parasitol. 116: 87-95.

36. Osaer, S., B. Goossens, S. Kora, et al. 1999. Health and productivity of traditionally managed Djallonke sheep and West African dwarf goats under high and moderate trypanosomosis risk. Vet. Parasitol. 82: 101-119.

37. Chiejina, S.N., G.A. Musongong, B.B. Fakae, et al. 2005. The modulatory influence of Trypanosoma brucei on challenge infection with Haemonchus contortus in Nigerian West African Dwarf goats segregated into weak and strong responders to the nematode. Vet. Parasitol. 128: 29-40.

38. Diesing, L., J.S. Ahmed, E. Zweygarth, et al. 1983. Trypanosoma brucei brucei infection in goats. Response in peripheral blood lymphocytes to mitogen stimulation. Tropenmed. Parasitol. 34: 79-83.

39. MAKUMYAVIRI, A.M. 1991. Epidemiological importance of the experimental host and of the animal reservoir of Trypanosoma brucei gambiense. Rev. Med. Vet. 141: $873-875$.

40. Robson, J. \& L.R. Rickman. 1973. Blood incubation infectivity test results for Trypanosoma brucei subgroup isolated in the Lambwe Valley, South Nyanza, Kenya. Trop. Anim. Health Prod. 5: 187-191.

41. DavilA, A.M.R. \& R.A.M.S. Silva. 2000. Animal trypanosomiasis in South America. Current status, Partnership, and information technology. Ann. N. Y. Acad. Sci. 916: 199-212.

42. GARdiner, P.R. \& M.M. MAHMoud. 1992. Salivarian trypanosomes causing disease in livestock outside Sub-Saharan Africa. In Parasitic Protozoa. J.P. Kreier \& J.R. Baker, Eds.: Vol. 2: 277-314. Academic Press. San Diego, California.

43. VAN DAM, R.H. et al. 1981. Trypanosome mediated suppression of humoral and cell-mediated immunity in goats. Vet. Parasitol. 8: 1-11.

44. VAn Den Ingh, T.S.G.A.M., D. Zwart, A.J. Schotman, et al. 1976. The pathology and pathogenesis of Tripanosoma vivax in the goat. Res. Vet. Sci. 21: 264-270.

45. VeenendaAl, G.H., A.S. VAn Miert, T.S. Van Den Ingh, et al. 1976. A comparison of the role of kinins and serotonin in endotoxin induced fever and Trypanosoma vivax infections in the goat. Res. Vet. Sci. 21: 271-279.

46. Herrera, L., P.S. DE' Andrea, S.C.C. XAVIER, et al. Trypanosoma cruzi infection in wild mammals of the National Park "Serra da Capibara" and its surroundings (Piauí, Brazil), an area endemic for Chagas disease. Trans. Royal Soc. Trop. Med. Hyg. 99: 379-388.

47. Diamond, L.S. \& R. Rubin. 1958. Experimental infection of certain farm animals with a North American strain of Trypanosoma cruzi from the raccoon. Exp. Parasitol. 7: 383-390. 